\title{
Nonlinear propagation in Kerr media of beams with unequal transverse widths
}

\author{
B. Crosignani and P. Di Porto \\ Dipartimento di Fisica, Università dell'Aquila, 67010 L'Aquila, Italy
}

Received January 25, 1993

\begin{abstract}
We investigate three-dimensional propagation in bulk Kerr media of beams possessing different widths in the two transverse dimensions, under the combined effects of self-focusing and diffraction. This allows us to elucidate the process of self-trapping and provides a quantitative interpretation of recent experimental observations of beam self-deflection [Barthelemy et al., Opt. Lett. 17, 844 (1992)].
\end{abstract}

Much attention has recently been devoted, both theoretically ${ }^{1-5}$ and experimentally, ${ }^{6-11}$ to the investigation of self-trapped propagation of optical beams (spatial solitons), which can be achieved as a result of balance between self-focusing and diffraction in Kerr media. In the three-dimensional case (propagation along $z$ and diffraction along the transverse directions $x$ and $y$ ), self-trapping is unstable under radially symmetric perturbations of the circularly symmetric transverse configuration, ${ }^{12}$ while the analysis of the stability properties becomes more involved for asymmetric perturbations..$^{13}$

The three-dimensional case degenerates into the (stable) two-dimensional one if circular symmetry is removed by letting one of the two transverse widths of the beam (e.g., $y$ ) become large enough that the corresponding diffraction length exceeds the propagation length. In this situation, the $y$ dimension is practically infinite and the significant dynamical evolution is restricted to the $x$ dimension. However, the propagation length can be such that the nonlinearity associated with the optical Kerr effect makes itself manifest through an experimentally observable beam self-deflection, ${ }^{14}$ so that it becomes necessary to investigate the beam behavior without neglecting its evolution along the $y$ dimension.

In this Letter we use a general formalism based on a variational approach to describe propagation of beams with different transverse widths. This allows us, in particular, to interpret the main features of the self-deflection experiment described in Ref. 14.

We recall that stationary three-dimensional propagation is described by the dimensionless nonlinear Schrödinger equation, ${ }^{12}$

$$
i \frac{\partial}{\partial \zeta} u+\frac{1}{2}\left(\frac{\partial^{2}}{\partial \xi^{2}}+\frac{\partial^{2}}{\partial \eta^{2}}\right) u+|u|^{2} u=0
$$

where $(\xi, \eta, \zeta)=(k x, k y, k z)$ and $u=\left(n_{2} / n_{0}\right)^{1 / 2} E$, with $n_{2}$ being the nonlinear refractive-index coefficient of the medium (whose refractive index is given by $n=n_{0}+n_{2}|E|^{2}$ ) and $E$ labeling the slowly varying part of the electric field $\exp (i k z-i \omega t) E(x, y, z)$. Equation (1) can be interpreted as a Euler-Lagrange equation ${ }^{15}$ corresponding to a vanishing variation

$$
\begin{aligned}
\delta \int_{-\infty}^{+\infty} \mathrm{d} \xi \int_{-\infty}^{+\infty} \mathrm{d} \eta \int_{-\infty}^{+\infty} \mathrm{d} \zeta \\
\quad \times \mathcal{L}\left(u, u^{*}, u_{\xi}, u_{\xi}{ }^{*}, u_{\eta}, u_{\eta}{ }^{*}, u_{\zeta}, u_{\zeta}{ }^{*}\right)=0
\end{aligned}
$$

of the Lagrangian density

$$
\mathcal{L}=\frac{1}{2 i}\left(u^{*} u_{\zeta}-u u_{\zeta}^{*}\right)+\frac{1}{2}\left|u_{\xi}\right|^{2}+\frac{1}{2}\left|u_{\eta}\right|^{2}-\frac{1}{2}|u|^{4},
$$

where the subscripts denote partial derivative.

In the context of the Ritz optimization procedure, as used in the frame of optical propagation problems, ${ }^{3,4,13}$ we look for an approximate analytical solution of Eq. (1) to be found within a set of suitably chosen trial functions of the form

$$
\begin{aligned}
u(\xi, \eta, \zeta)= & \frac{M_{0}^{1 / 2}(\zeta)}{\sigma^{1 / 2}(\zeta) \mu^{1 / 2}(\zeta)} F^{1 / 2}\left(\frac{\xi^{2}}{\sigma^{2}}+\frac{\eta^{2}}{\mu^{2}}\right) \\
& \times \exp \left[i a_{0}(\zeta)+i a_{1}(\zeta) \xi^{2}+i a_{2}(\zeta) \eta^{2}\right],
\end{aligned}
$$

where $F$ is a well-behaved prescribed function and the two widths $\sigma$ and $\mu$ (along the $x$ and $y$ directions, respectively) are permitted to assume different values. If we insert Eq. (4) into Eq. (2), the variational principle reduces to

$$
\delta \int_{-\infty}^{+\infty} \mathrm{d} \zeta \mathcal{L}_{r}=0
$$

with

$$
\mathcal{L}_{r}=\int_{-\infty}^{+\infty} \mathrm{d} \xi \int_{-\infty}^{+\infty} \mathrm{d} \eta \mathcal{L} .
$$

Accordingly, the exact field Lagrangian $\mathcal{L}$ is replaced by the reduced Lagrangian $\mathcal{L}_{r}$, whose Euler-Lagrange equations furnish the evolution of the $\zeta$-dependent parameters $M_{0}, \sigma, \mu, a_{0}, a_{1}$, and $a_{2}$. We wish to consider here the situation in which $|u(\zeta=0, \xi, \eta)|$ has a Gaussian shape and to assume that the trial function $F$ maintains a Gaussian distribution in both $\xi$ and $\eta$ for varying $\zeta$, that is,

$$
\begin{aligned}
& u(\xi, \eta, \zeta)=\frac{1}{\sqrt{\pi}} \frac{M_{0}^{1 / 2}}{\sigma^{1 / 2}(\zeta) \mu^{1 / 2}(\zeta)} \exp \left\{-\left[\xi^{2} / 2 \sigma^{2}(\zeta)\right]\right. \\
& \left.-\left[\eta^{2} / 2 \mu^{2}(\zeta)\right]\right\} \exp \left[i a_{0}(\zeta)+i a_{1}(\zeta) \xi^{2}+i a_{2}(\zeta) \eta^{2}\right] .
\end{aligned}
$$


After inserting Eq. (7) into Eq. (3) and the resulting expression into Eq. (6), we obtain

$$
\begin{aligned}
\mathcal{L}_{r}= & \dot{a}_{0} M_{0}+\frac{1}{2} M_{0}\left(\dot{a}_{1} \sigma^{2}+\dot{a}_{2} \mu^{2}\right)-\frac{1}{4 \pi} \frac{M_{0}^{2}}{\sigma \mu} \\
& +M_{0}\left(\sigma^{2}{a_{1}}^{2}+\mu^{2} a_{2}^{2}\right)+\frac{M_{0}}{4}\left(\frac{1}{\sigma^{2}}+\frac{1}{\mu^{2}}\right),
\end{aligned}
$$

where the overdot denotes derivation with respect to $\zeta$. The Euler-Lagrange equations pertinent to the above Lagrangian read as (we omit, for conciseness, the one describing the evolution of $a_{0}$ since it is not relevant for the results of this Letter)

$$
\begin{gathered}
\dot{M}_{0}=0, \\
\dot{\sigma}-2 \sigma a_{1}=0, \quad \dot{\mu}-2 \mu a_{2}=0 \\
\dot{a}_{1}+\frac{1}{4 \pi} \frac{M_{0}}{\sigma^{3} \mu}+2{a_{1}}^{2}-\frac{1}{2 \sigma^{4}}=0 \\
\dot{a}_{2}+\frac{1}{4 \pi} \frac{M_{0}}{\sigma \mu^{3}}+2{a_{2}}^{2}-\frac{1}{2 \mu^{4}}=0 .
\end{gathered}
$$

By differentiating Eqs. (10) with respect to $\zeta$, we obtain, with the help of Eqs. (11), (12), and (9) (which expresses energy conservation),

$$
\begin{aligned}
& \ddot{\sigma}=-\frac{1}{2 \pi} \frac{M_{0}}{\sigma^{2} \mu}+\frac{1}{\sigma^{3}}, \\
& \ddot{\mu}=-\frac{1}{2 \pi} \frac{M_{0}}{\sigma \mu^{2}}+\frac{1}{\mu^{3}} .
\end{aligned}
$$

The set of Eqs. (13) and (14) describes the $\zeta$ evolution of an asymmetric Gaussian beam, the asymmetry being associated with nonidentical values of the initial widths along the $x$ and $y$ axes, that is, $\sigma(\zeta=0) \equiv \sigma_{0} \neq \mu(\zeta=0) \equiv \mu_{0}$.

The usual two-dimensional situation corresponds to the assumption that both nonlinearity and diffraction are ineffective in the $y$ direction, which amounts to letting $\mu$ go to infinity in Eq. (14) and setting $\mu=\mu_{0}$ in Eq. (13). The resulting equation,

$$
\ddot{\sigma}=-\frac{1}{2 \pi} \frac{M_{0}}{\sigma^{2} \mu_{0}}+\frac{1}{\sigma^{3}},
$$

exhibits both the nonlinear Kerr contribution (the term proportional to the energy $M_{0}$ ) and the diffractive contribution (the term proportional to $1 / \sigma^{3}$ ), and the behavior of its solutions can be investigated by noting that $2 \mathrm{~d}^{2} \sigma / \mathrm{d} \zeta^{2}=(\mathrm{d} / \mathrm{d} \sigma)\left[(\mathrm{d} \sigma / \mathrm{d} \zeta)^{2}\right]$. If we assume that $(\mathrm{d} \sigma / \mathrm{d} \zeta)_{\zeta=0}=0$ (corresponding to the beam waist in the input plane $z=0$ ), we obtain

$$
\sigma \frac{\mathrm{d} \sigma}{\mathrm{d} \zeta}=\frac{1}{\sqrt{2} \sigma_{0}}\left[\left(\sigma-\sigma_{0}\right)\left(\sigma_{0}-\alpha \sigma\right)\right]^{1 / 2}
$$

where

$$
\alpha=\frac{1}{\pi} \frac{M_{0} \sigma_{0}}{\mu_{0}}-1 .
$$

Different propagation regimes turn out to exist according to the values assumed by the parameter $\alpha$. If $\alpha=1$, that is, for the critical value of the power $M_{0}=\left\langle M_{0}\right\rangle=2 \pi\left(\mu_{0} / \sigma_{0}\right)$, Eqs. (15) and (16) are consistent with the self-trapped solution $\sigma(\zeta)=\sigma_{0}$ (spatial soliton). If $-1<\alpha \leq 0$, one has a diffractiondominated regime in which $\sigma(\zeta) \rightarrow \infty$ for $\zeta \rightarrow \infty$. For $0<\alpha<1, \sigma(\zeta)$ is a periodic function whose value oscillates from $\sigma_{0}$ to $\sigma_{0} / \alpha>\sigma_{0}$, whereas for $\alpha>1, \sigma(\zeta)$ is a periodic function oscillating from $\sigma_{0}$ to $\sigma_{0} / \alpha<\sigma_{0}$. In practice, for $\alpha$ slightly different from unity, the beam behaves like a spatial soliton whose width undergoes small oscillations around the equilibrium value $\sigma_{0}$ corresponding to $\alpha=1$, which expresses the well-known stability properties of twodimensional solitons.

We now address the situation pertaining to beam self-deflection experiments, ${ }^{14}$ in which $\sigma$ can be considered approximately constant over the relevant propagation length, while the evolution of $\mu(\zeta)$ is expedient for the understanding of the self-deflection process. Actually, the experiment of Ref. 14 deals with nonlinear propagation of a beam with an elliptical transverse section (whose major axis corresponds to the $y$ axis). This pattern is obtained by the superposition of two tilted beams, which gives rise to a system of interference fringes (parallel to the $x$ axis) whose role is to prevent filamentation without essentially influencing the evolution of the transverse beam shape. The authors of Ref. 14 interpret the nonlinear dynamics affecting diffraction in the $y$ direction in terms of attraction of the two interfering beams, which they call self-deflection. In our formalism, this attraction corresponds to an increasing of $\mu$ with $\zeta$ slower than that associated with diffraction alone [see relation (22) below].

To describe the evolution of $\mu(\zeta)$, it is necessary to solve Eq. (14), which we rewrite in the approximate form

$$
\ddot{\mu}=-\frac{1}{2 \pi} \frac{M_{0}}{\sigma_{0} \mu^{2}} .
$$

Equation (18) corresponds to neglecting the $\zeta$ dependence of the small dimension $\sigma$ as well as diffraction effects along the large dimension $\mu(\zeta)$, an approximation valid under the experimental conditions of Ref. 14. By use of the relation $2 \mathrm{~d}^{2} \mu / \mathrm{d} \zeta^{2}=$ $(\mathrm{d} / \mathrm{d} \mu)\left[(\mathrm{d} \mu / \mathrm{d} \zeta)^{2}\right]$, Eq. (18) gives rise to

$$
\frac{\mathrm{d} \mu}{\mathrm{d} \zeta}=\left[\gamma\left(\frac{1}{\mu}-\frac{1}{\mu_{0}}\right)+q_{0}^{2}\right]^{1 / 2},
$$

where $q_{0}=(\mathrm{d} \mu / \mathrm{d} \zeta)_{\zeta=0}$ and $\gamma=M_{0} / \pi \sigma_{0}$. For the power levels of Ref. 14 , one has $\beta \equiv 1 / \mu_{0}-q_{0}^{2} / \gamma>0$, and Eq. (19) is easily integrated to give

$$
\begin{aligned}
& \arccos \left\{[\beta \mu(\zeta)]^{1 / 2}\right\}+\{[\beta \mu(\zeta)][1-\beta \mu(\zeta)]\}^{1 / 2} \\
& \quad-\arccos \left[\left(\beta \mu_{0}\right)^{1 / 2}\right]-\left[\beta \mu_{0}\left(1-\beta \mu_{0}\right)\right]^{1 / 2}=-\beta^{3 / 2} \gamma^{1 / 2} \zeta .
\end{aligned}
$$

To obtain an explicit expression for $\mu(\zeta)$, we expand the left-hand side of Eq. (20) around $\mu=\mu_{0}$, thus getting

$$
\left(\mu-\mu_{0}\right)+\frac{\gamma}{4 \mu_{0}^{2} q_{0}^{2}}\left(\mu-\mu_{0}\right)^{2}=q_{0} \zeta,
$$


from which it is easily deduced that

$$
\frac{\mathrm{d} \mu}{\mathrm{d} \zeta}=\frac{q_{0}}{1+\frac{\gamma\left(\mu-\mu_{0}\right)}{2 \mu_{0}^{2} q_{0}^{2}}} \cong q_{0}-\frac{\gamma}{2 \mu_{0}^{2}} \zeta .
$$

We now observe that, since $\mu$ represents the beam width in the $y$ direction, the quantity $\mathrm{d} \mu / \mathrm{d} \zeta$ can be interpreted as the angular divergence $\theta(\zeta)$ of the beam and the self-deflection effect is contained in the negative $\zeta$-dependent term on the right-hand side of relation (22). In ordinary units, relation (22) yields

$$
\theta(z)=\theta(0)-\frac{n_{2} Z_{0} I}{2 \pi n_{0}}\left(z / w_{y}\right)
$$

where $Z_{0}$ is the vacuum impedance and $I=P /\left(w_{x} w_{y}\right)$ is the beam intensity, with $P$ the power and $w_{x}=$ $\sigma_{0} / k$ and $w_{y}=\mu_{0} / k$ the beam widths. In the experiment of Ref. 14, the nonlinear medium is $\mathrm{CS}_{2}$, for which $n_{0}=1.6$ and $n_{2}=1.9 \times 10^{-20}(\mathrm{~m} / \mathrm{V})^{2}$, and the beam has an elliptical transverse shape with $w_{x}=60 \mu \mathrm{m}$ and $w_{y}=4 \mathrm{~mm}$. The measured self-deflection $\left(0.6 \mathrm{mrad}\right.$ for $\left.I \cong 5 \mathrm{GW} / \mathrm{cm}^{2}\right)$ can be compared with the theoretical value of $0.46 \mathrm{mrad}$ given by Eq. (23).

In conclusion, we have developed a formalism that allows us to describe the nonlinear propagation of beams with unequal transverse widths. It is based on a variational approach already well established in the framework of nonlinear optics, which has been shown to provide analytical results in good agreement with the numerical ones. ${ }^{3,4,16}$ In particular, we have presented an application in connection with recent observations of self-deflection in Kerr media. ${ }^{14}$

This research has been partially supported by the Consorzio Interuniversitario Nazionale di Fisica della
Materia del Ministero dell'Università e della Ricerca Scientifica e Tecnologica.

\section{References}

1. A. W. Synder, D. J. Mitchell, L. Poladian, and L. Ladouceur, Opt. Lett. 16, 21 (1991).

2. Q. Y. Li, C. Pask, and R. A. Sammut, Opt. Lett. 16, 1083 (1991).

3. M. Karisson, D. Anderson, M. Desaix, and M. Lisak, Opt. Lett. 16, 1373 (1991).

4. M. Desaix, D. Anderson, and M. Lisak, J. Opt. Soc. Am. B 8, 2082 (1991).

5. J. T. Manassah, Opt. Lett. 17, 1259 (1992).

6. A. Barthelemy, S. Maneuf, and C. Froehly, Opt. Commun. 55, 201 (1985).

7. S. Maneuf, R. Desailly, and C. Froehly, Opt. Commun. 65, 193 (1985).

8. S. Maneuf and F. Reynaud, Opt. Commun. 66, 325 (1988).

9. F. Reynaud and A. Barthelemy, Europhys. Lett. 12, 401 (1990).

10. J. S. Aitchison, A. M. Weiner, Y. Silberberg, M. K. Oliver, J. L. Jackel, D. E. Leaird, E. M. Vogel, and P. W. E. Smith, Opt. Lett. 15, 471 (1990).

11. J. S. Aitchison, A. M. Weiner, Y. Silberberg, D. E. Leaird, M. K. Oliver, J. L. Jackel, and P. W. E. Smith, Opt. Lett. 16, 15 (1991).

12. Y. Silberberg, Opt. Lett. 15, 1282 (1990).

13. B. Crosignani, P. Di Porto, and S. Piazzolla, Pure Appl. Opt. 1, 7 (1992).

14. A. Barthelemy, C. Froehly, S. Maneuf, and F. Reynaud, Opt. Lett. 17, 844 (1992).

15. H. Goldstein, Classical Mechanics, 2nd ed. (AddisonWesley, Reading, Mass., 1980), Chap. 12, pp. 548-552.

16. B. Crosignani and P. Di Porto, Opt. Commun. 89, 453 (1992). 